\title{
Rules Ventral Prefrontal Cortical Axons Use to Reach Their Targets: Implications for Diffusion Tensor Imaging Tractography and Deep Brain Stimulation for Psychiatric Illness
}

\author{
Julia F. Lehman, ${ }^{1}$ Benjamin D. Greenberg, ${ }^{2}$ Cameron C. McIntyre, ${ }^{3}$ Steve A. Rasmussen, ${ }^{2}$ and Suzanne N. Haber ${ }^{1}$ \\ ${ }^{1}$ Department of Pharmacology and Physiology, University of Rochester School of Medicine, Rochester, New York 14642, ${ }^{2}$ Department of Psychiatry, Butler \\ Hospital and Brown University, Providence, Rhode Island 02912, and ${ }^{3}$ Department of Biomedical Engineering, Cleveland Clinic Foundation, Cleveland, \\ Ohio 44195
}

The ventral prefrontal cortex (vPFC) is involved in reinforcement-based learning and is associated with depression, obsessivecompulsive disorder, and addiction. Neuroimaging is increasingly used to develop models of vPFC connections, to examine white matter (WM) integrity, and to target surgical interventions, including deep brain stimulation. We used primate (Macaca nemestrina/Macaca fascicularis) tracing studies and 3D reconstructions of WM tracts to delineate the rules vPFC projections follow to reach their targets. vPFC efferent axons travel through the uncinate fasciculus, connecting different $\mathrm{vPFC}$ regions and linking different functional regions. The uncinate fasciculus also is a conduit for vPFC fibers to reach other cortical bundles. Fibers in the internal capsule are organized according to destination. Thalamic fibers from each vPFC region travel dorsal to their brainstem fibers. The results show regional differences in the trajectories of fibers from different $\mathrm{vPFC}$ areas. Overall, the medial/lateral vPFC position dictates the route that fibers take to enter major WM tracts, as well as the position within specific tracts: axons from medial vPFC regions travel ventral to those from more lateral areas. This arrangement, coupled with dorsal/ventral organization of thalamic/brainstem fibers through the internal capsule, results in a complex mingling of thalamic and brainstem axons from different vPFC areas. Together, these data provide the foundation for dividing vPFC WM bundles into functional components and for predicting what is likely to be carried at different points through each bundle. These results also help determine the specific connections that are likely to be captured at different neurosurgical targets.

\section{Introduction}

The ventral prefrontal cortex (vPFC) is a complex area comprising multifunctional regions that interface with cortical and subcortical structures involved in reward processing, reinforcement-based learning, and decision making (O'Doherty et al., 2003; Chase et al., 2008; Rudebeck et al., 2008). vPFC dysfunction is associated with several psychiatric disorders (Volkow et al., 1999; Mayberg, 2007; Chamberlain et al., 2008). Insights into the neural networks that mediate these behaviors come from human and nonhuman primate connectivity experiments (Beckmann et al., 2009; Price and Drevets, 2010). Diffusion tensor imaging (DTI) is

\footnotetext{
Received Feb. 2, 2011; revised April 27, 2011; accepted May 25, 2011.

Author contributions: S.N.H. designed research; J.F.L. and S.N.H. performed research;J.F.L., B.D.G., C.C.M., S.A.R., and S.N.H. analyzed data; B.D.G., S.A.R., and S.N.H. wrote the paper.

C.C.M. is a paid consultant for Boston Scientific Neuromodulation Corp. and IntElect Medical Inc.

This work was supported by National Institute of Mental Health Grants MH73111 and MH086400 and National Institute of Neurological Disorders and Stroke Grant NS059736. We thank Ashutosh Chaturvedi and Angela M. Noecker for assistance with the stimulation calculations.

This article is freely available online through the J Neurosci Open Choice option.

Correspondence should be addressed to Dr. S. N. Haber, Department of Pharmacology and Physiology, University of Rochester School of Medicine, 601 Elmwood Avenue, Rochester, NY 14642. E-mail: suzanne_haber@urmc.rochester.edu. DOI:10.1523/JNEUROSCI.0595-11.2011

Copyright $\odot 2011$ the authors $\quad 0270-6474 / 11 / 3110392-11 \$ 15.00 / 0$
}

now widely used to develop connectivity profiles of the human vPFC and is a key tool for examining potential white matter (WM) pathology in psychiatric disorders and for targeting vPFC surgical interventions, including deep brain stimulation (DBS) (Croxson et al., 2005; Johansen-Berg et al., 2008; Price et al., 2008; Cullen et al., 2010). However, it is the anatomical data from nonhuman primates that provide the foundation for precisely defining and segmenting WM bundles into their components (Croxson et al., 2005; Makris et al., 2005; Schmahmann et al., 2007). Segmentation of the superior longitudinal fasciculus in humans, for example, was based on data from monkeys (Makris et al., 2005). Together, DTI and primate tracing studies constitute a powerful approach for defining the structural networks that underlie emotion, motivation, and cognition; for identifying specific pathways affected by WM pathology; and for guiding invasive neurosurgical procedures.

vPFC fibers reach cortical targets primarily via the uncinate fasciculus (UF) and extreme capsule (EmC). The UF occupies the ventral plate of the vPFC and connects PFC with the temporal lobe (Schmahmann and Pandya, 2006; Petrides and Pandya, 2007). The EmC lies between the insula and claustrum and carries association fibers between frontal, temporal, and parietal cortex. vPFC projections to subcortical regions travel primarily 
in the internal (IC) and external capsules (EC). Subcortical fibers pass through the EC to the ventral anterior limb of the IC (Beevor and Horsley, 1890; Schmahmann and Pandya, 2006; Petrides and Pandya, 2007). These major PFC pathways are well defined, but little is known about the organization of vPFC fibers within them. Of particular importance is determining whether fibers from different $\mathrm{vPFC}$ regions are segmented within these bundles and whether specific organizational rules can be identified. These data are fundamental for predicting where specific vPFC fibers should travel, thus, improving the accuracy of DTI tractography. In addition, identifying the rules and components of these pathways will lead to more precise identification of connections from fibers that are affected by pathology or targeted by invasive surgery (i.e., DBS).

Our experiments had three objectives: first, delineate relationships between pathways from different vPFC regions; second, determine organizational rules governing the trajectory of different vPFC efferent fiber bundles; and third, determine the specific pathways involved at the different DBS sites. The rationale for the last objective is that two of the most promising surgical sites for treating intractable depression and obsessive-compulsive disorder are within vPFC WM tracts (Mayberg et al., 2005; Greenberg et al., 2010b).

\section{Materials and Methods}

Overview. The $\mathrm{PPFC}$ was divided into four regions based on functional imaging studies (Petrides et al., 2002; Croxson et al., 2005). We injected anterograde or bidirectional tracers into the four regions in Old World primates: (1) ventral medial PFC (vmPFC; medial to the olfactory sulcus), (2) medial orbital cortex (mOFC; between the olfactory and medial orbital sulci), (3) central OFC orbital cortex (cOFC; between the medial and lateral orbital sulci), and (4) lateral orbital cortex (lOFC; at or lateral to the lateral orbital sulcus) (Fig. 1). Fiber bundles were charted and rendered in $3 \mathrm{D}$ for each experiment separately. They were then combined into one global 3D model. Representations of electrodes used in humans for the two WM DBS targets [the subgenual cingulate gyrus WM (SCGwm) and at the conjunction of the ventral internal capsule (VC) and ventral striatum (VS) (Hamani et al., 2009; Greenberg et al., 2010b)] were adjusted for size ( $40 \%$ of human dimensions) (Yin et al., 2009) and placed into the primate model. We also evaluated a third site centered in the nucleus accumbens but also involving part of the VS (Schlaepfer et al., 2008). Clinically relevant stimulation parameters were used to model stimulus spread for each contact at the VC/VS site (Miocinovic et al., 2007). Experiments were carried out in accordance with the Institute of Laboratory Animal Resources Guide for the Care and Use of Laboratory Animals and approved by the University Committee on Animal Resources.

Surgery and tissue preparation. Adult male macaque monkeys (Macaca nemestrina and Macaca fascicularis) were tranquilized by intramuscular injection of ketamine $(10 \mathrm{mg} / \mathrm{kg})$. A surgical plane of anesthesia was maintained by intravenous injection of pentobarbital (initial dose 20 $\mathrm{mg} / \mathrm{kg}$, i.v., and maintained as needed). Temperature, heart rate, and respiration were monitored throughout the surgery. Monkeys were placed in a Kopf stereotaxic, a midline scalp incision was made, and the muscle and fascia were displaced laterally to expose the skull. A craniotomy $\left(\sim 2-3 \mathrm{~cm}^{2}\right)$ was made over the region of interest and small dural incisions were made only at injection sites. MRI ( 3 tesla) T2 turbo spin echo scans $(0.5$ $\mathrm{mm} \times 0.5 \mathrm{~mm} \times 1.42 \mathrm{~mm}$ ) were obtained before surgery. These images were used to calculate the anterior/posterior, dorsal/ventral, and medial/ lateral coordinates for each tracer injection from stereotaxic zero.

Monkeys received an injection of one or more of the following anterograde/bidirectional tracers: Lucifer yellow, fluororuby, or fluorescein conjugated to dextran amine (LY, FR, or FS; $40-50 \mathrm{nl}, 10 \%$ in $0.1 \mathrm{M}$ phosphate buffer (PB), pH 7.4; Invitrogen); Phaseolus vulgarisleucoagglutinin (PHA-L; $50 \mathrm{nl}, 2.5 \%$; Vector Laboratories); or tritiated amino acids $\left(100 \mathrm{nl}, 1: 1\right.$ solution of $\left[{ }^{3} \mathrm{H}\right]$ leucine and $\left[{ }^{3} \mathrm{H}\right]$-proline in
$\left.\mathrm{dH}_{2} \mathrm{O}, 200 \mathrm{mCi} / \mathrm{ml}, \mathrm{NEN}\right)$. Tracers were pressure-injected over $10 \mathrm{~min}$ using a $0.5 \mu \mathrm{l}$ Hamilton syringe. Following each injection, the syringe remained in situ for 20-30 min. Twelve to $14 \mathrm{~d}$ after surgery, monkeys were again deeply anesthetized and perfused with saline followed by a $4 \%$ paraformaldehyde $/ 1.5 \%$ sucrose solution in $0.1 \mathrm{M} \mathrm{PB}, \mathrm{pH}$ 7.4. Brains were postfixed overnight and cryoprotected in increasing gradients of sucrose $(10 \%, 20 \%$, and $30 \%)$. Serial sections of $50 \mu \mathrm{m}$ were cut on a freezing microtome into $0.1 \mathrm{M}$ PB or cryoprotectant solution as described previously (Haber et al., 2006).

One in eight sections were processed free-floating for immunocytochemistry to visualize the tracers. Tissue was incubated in primary anti-LY (1:3000 dilution; Invitrogen), anti-FS (1:1000; Invitrogen), anti-FR (1:1000; Invitrogen), or anti-PHA-L (1:500; EY Laboratories) in $10 \%$ NGS and $0.3 \%$ Triton X-100 (Sigma-Aldrich) in PB for 4 nights at $4^{\circ} \mathrm{C}$. Following extensive rinsing, the tissue was incubated in biotinylated secondary antibody followed by incubation with the avidin-biotin complex solution (Vectastain ABC kit, Vector Laboratories). Immunoreactivity was visualized using standard $\mathrm{DAB}$ procedures. Staining was intensified by incubating the tissue for $5-15 \mathrm{~s}$ in a solution of $0.05 \%$ 3,3'-diaminobenzidine tetra-hydrochloride, $0.025 \%$ cobalt chloride, $0.02 \%$ nickel ammonium sulfate, and $0.01 \% \mathrm{H}_{2} \mathrm{O}_{2}$. Sections were mounted onto gel-coated slides, dehydrated, defatted in xylene, and coverslipped with Permount. In cases in which more than one tracer was injected into a single animal, adjacent sections were processed for each antibody reaction. Sections for autoradiography were mounted on chrome-alum gelatin-coated slides and defatted in xylene overnight. Slides were dipped in Kodak NTB2 photographic emulsion and exposed for $4-6$ months at $4^{\circ} \mathrm{C}$ in a light-tight box. The sections were then developed in Kodak D19 for 2.5 min., fixed, washed, and counterstained with cresyl violet.

Data analysis. All thick fibers without clear terminal boutons were assumed to be passing fibers and were included in the analysis. Fibers traveling in bundles were outlined as a group. Orientation was indicated for each bundle by charting a few individual fibers within each outline. Fibers outside of the major bundles were traced individually. There were seven injections into different regions of the vmPFC, three into the $\mathrm{mOFC}$, five in the COFC, and four into the lOFC. One animal received three different tracer injections placed into different regions of $\mathrm{vPFC}$ (vmPFC, cOFC, and lOFC).

$3 D$ reconstructions. $3 \mathrm{D}$ renderings of the pathways were developed in the following way. Fiber bundles were outlined using a 4.0 or 6.4 objective and Neurolucida software (MBF Bioscience). Some individual fibers within each bundle were charted at higher magnification $(10 \times)$ to indicate directionality. For each case, a stack of $2 \mathrm{D}$ coronal sections was created from its Neurolucida chartings and Nissl-stained images. This stack was imported into IMOD (Boulder Laboratory for 3D Electron Microscopy) (Kremer et al., 1996), and a 3D reconstruction that contained the fiber bundles was created for each case separately. To merge several cases together, we developed a reference model from one animal by sampling one in four sections (at $200 \mu \mathrm{m}$ intervals) throughout the entire brain, using frozen and Nissl-stained sections. Data from each case were then transposed into the reference brain using landmarks of key internal structures. Following the transposition of projections from each case, every contour placed in the reference model was checked with the original case for medial/lateral, dorsal/ventral, and anterior/posterior placement and its relative size. This assured that each of the fiber bundles from the different cases was accurately placed with respect to its position and the proportion of the WM tract it occupied. Thus, a 3D rendering was created first for each single case and then for the combinations of cases (Haber et al., 2006). Verification of the relationships between fibers bundles from different $\mathrm{vPFC}$ regions was demonstrated with multiple injections into the different $\mathrm{vPFC}$ regions in a single animal.

Neurostimulation modeling. We used the proportional distance between the length of the internal capsule from its dorsal boundary with the subcortical WM to the anterior commissure (AC) between the monkey and human brain, which is $\sim 40 \%$ and similar to proportional volume of the monkey striatum (Yin et al., 2009), to scale the electrode size and contact points. This electrode size and spacing of stimulation sites place each of the sites in approximately the same position in the monkey brain 

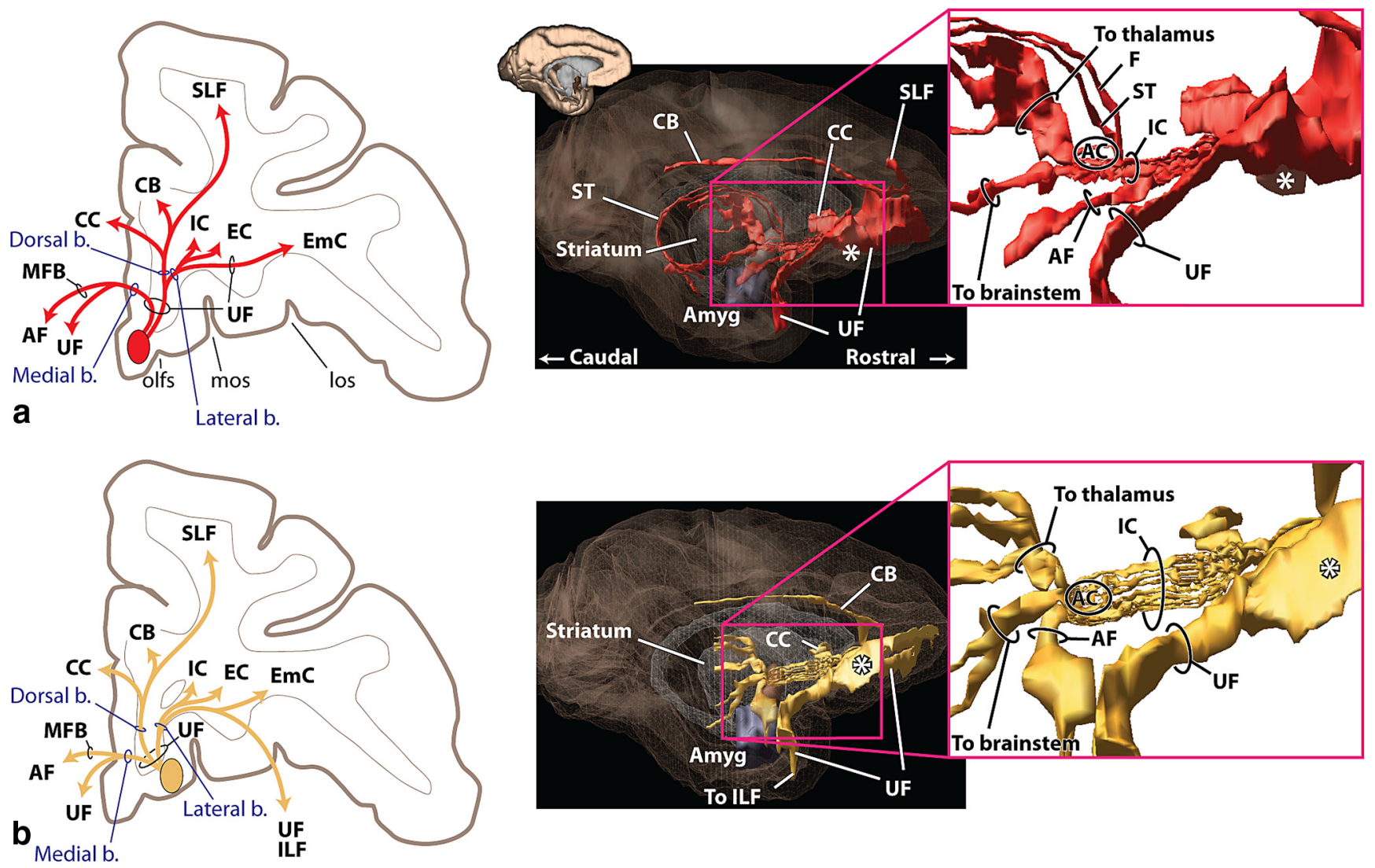
Medial b.

ILF
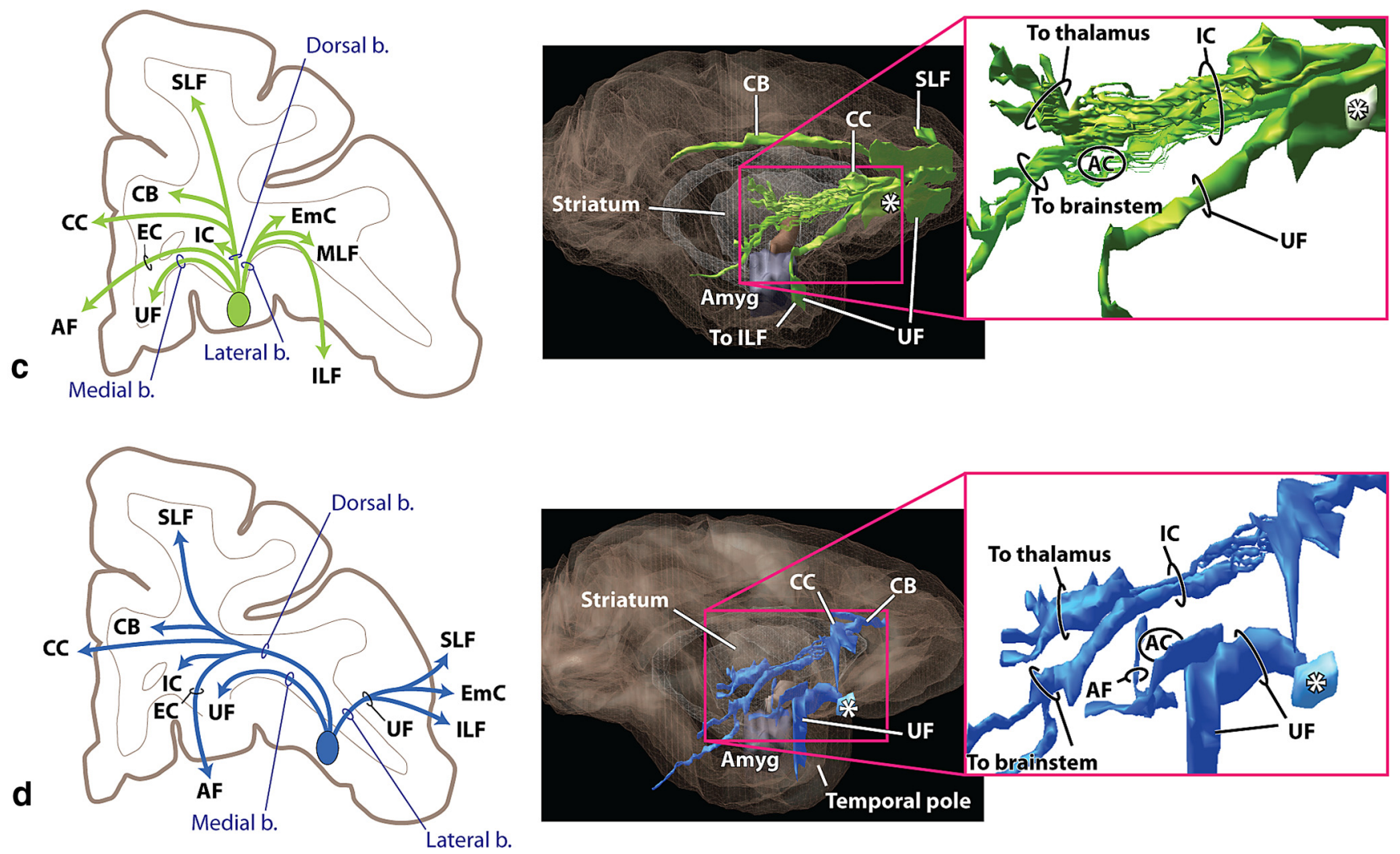

Figure 1. vPFC fiber pathways. $\boldsymbol{a}, \mathrm{vmPFC} . \boldsymbol{b}, \mathrm{mOFC} . \boldsymbol{c}, \mathrm{COFC} . \boldsymbol{d}$, IOFC. Left panels illustrate how different bundles separate from the injection site as they enter the white matter. Note, in each case, fibers divide into medial, dorsal, and lateral pathways (blue). However, the specific bundles that are carried within each depend on the position of the origin of the fibers. Right panels illustrate 3D renderings of a lateral view of a sagittal plane. Each is accompanied with an inset to better visualize the separation of fiber bundles. External and extreme capsule pathways have been removed for clarity. Asterisks indicate the rostrocaudal position of the injection site. AC indicates location of the anterior commissure. Axons from the vPFC, m0FC, and cOFC travel ventral or through the AC. Note that axons traveling through the internal capsule divide into dorsal thalamic fibers and ventral brainstem axons. Pathways traveling to the temporal lobe (Figure legend continues.) 

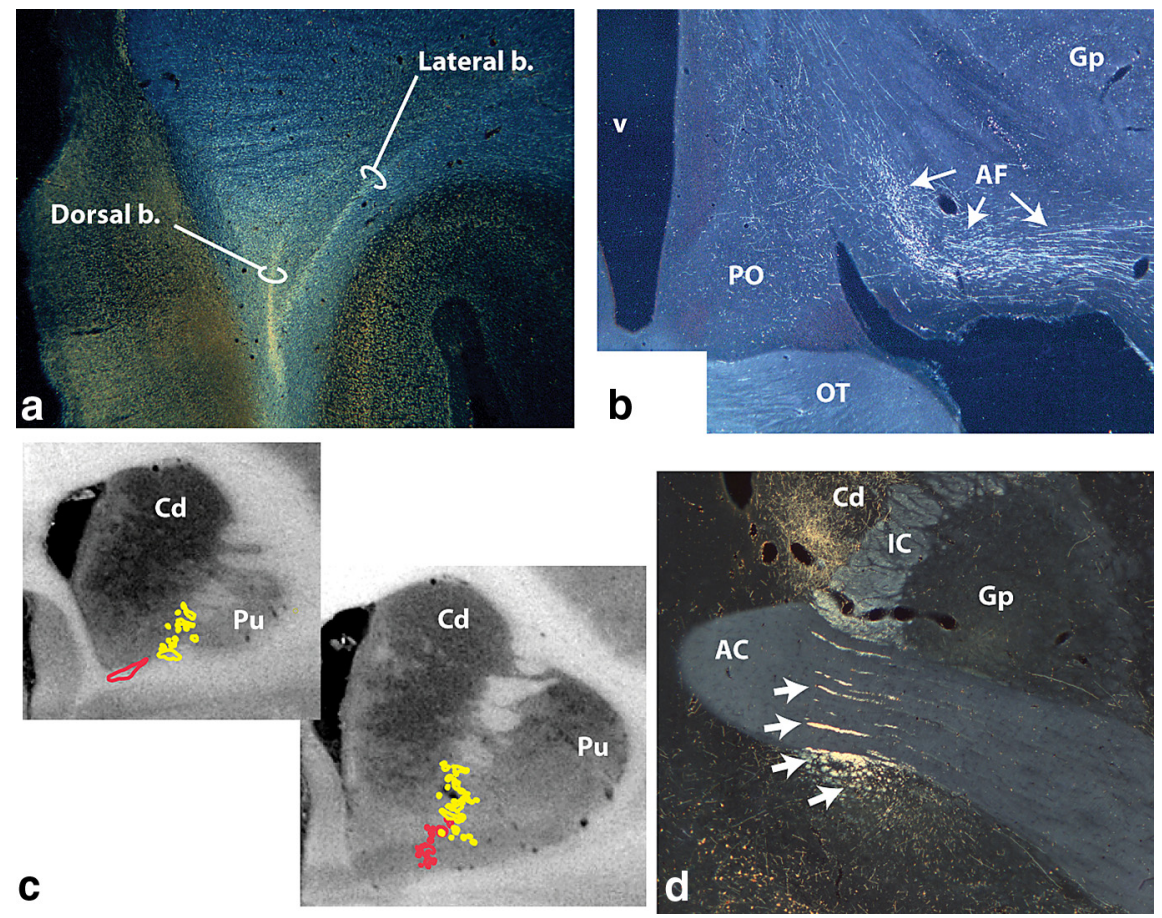

Figure 2. Photomicrographs and schematics of vmPFC and mOFC pathways. $\boldsymbol{a}$, vmPFC axons leave the injection site traveling dorsally and divide into the dorsal and lateral bundles. Some dorsal fibers enter the emerging corpus callosum. The medial bundle remains ventral (not illustrated). $\boldsymbol{b}$, The ventral amygdalofugal pathway carries fibers from the vmPFC to the amygdala. $\boldsymbol{c}$, Fibers from the vmPFC (red) and m0FC (yellow) enter the IC ventrally and form fascicules within the ventral striatum. $\boldsymbol{d}$, Photomicrograph illustrating $\mathrm{mOFC} \mathrm{IC} \mathrm{axons} \mathrm{embedded} \mathrm{within} \mathrm{the} \mathrm{AC}$ or ventral to it (arrows). Those within the IC terminate in the thalamus; the ventral groups continue to the brainstem. $\mathrm{AC}$, Anterior commissure; $\mathrm{AF}$, ventral amygdalofugal pathway; $b$., bundle; $\mathrm{Cd}$, caudate nucleus; Gp, globus pallidus; IC, internal capsule; $0 T$, optic tract; $P 0$, preoptic area; $P$ u, putamen; $v$, ventricle.

as described in the literature for human placement, including the subgenual WM site (Schlaepfer et al., 2008; Hamani et al., 2009; Greenberg et al., 2010b).

Coregistered histological slices and 3D reconstructions of the axonal pathways were imported into a customized version of Monkey Cicerone (Miocinovic et al., 2007). We created a database of the volume of tissue activated (VTA) for the four contacts scaled version of the DBS electrode ( $0.47 \mathrm{~mm}$ diameter, $0.56 \mathrm{~mm}$ contact height, $0.56 \mathrm{~mm}$ contact spacing) by simulating axonal activation thresholds via the combination of a finite element electric field model (FEM) and multicompartment cable models. The axisymmetric FEM of the scaled DBS electrode included a 57\% voltage drop at the electrode-electrolyte interface, an electrode capacitance of $0.6 \mu \mathrm{F}$, a $0.25-\mathrm{mm}$-thick sheath of encapsulation tissue $(0.18$ $\mathrm{S} / \mathrm{m}$ ), and $0.2 \mathrm{~S} / \mathrm{m}$ bulk tissue conductivity, with boundaries $7 \mathrm{~cm}$ from the electrode. Multicompartment myelinated axon models $(5.7 \mu \mathrm{m})$ were distributed with $0.05 \mathrm{~mm}$ spacing in a square grid around the DBS electrode (7991 total axons). The voltage distribution generated in the tissue medium by the DBS electrode, as calculated from the FEM, was applied to each axon model. The extracellular voltages applied to the axons generated inward and outward current flow that under the appropriate conditions could result in action potential initiation. Activation thresholds (DBS voltage necessary to produce a propagating action potential in each axon model) were calculated and the results were used to define contours representing

(Figure legend continued.) (uncinate fasciculus and ventral amygdalofugal bundle) primarily arise from separate bundles. AF, Ventral amygdalofugal pathway; Amyg, amygdala; b., bundle; $\mathrm{CB}$, cingulum bundle; $\mathrm{CC}$, corpus callosum; $\mathrm{EC}$, external capsule; $\mathrm{EmC}$, extreme capsule; $F$, fornix; IC, internal capsule; ILF, inferior longitudinal fasciculus; los, lateral orbital sulcus; MFB, medial forebrain bundle; MLF, middle longitudinal fasciculus; mos, medial orbital sulcus; olfs, olfactory sulcus; SLF, superior longitudinal fasciculus; ST, stria terminalis; UF, uncinate fasciculus; *injection site location.
VTAs for stimuli of various amplitudes (1-10 V) and pulse widths $(60,90,120 \mu \mathrm{s})$.

\section{Results}

All vPFC axons enter the UF immediately adjacent to their cortical region. Within the UF, fiber bundles from each injection site split into three groups, a medial, dorsal, and lateral group. Each of these groups of fibers contains subsets of axons that travel in different WM tracts, the specifics of which depend on the location of the injection site (Figs. 1, 2a, 3a). Axons from all vPFC areas travel in the UF, corpus callosum, cingulum bundle, superior longitudinal fasciculus, IC, EC, and EmC. In addition, fibers from specific vPFC regions also travel in the middle longitudinal fasciculus, ventral amygdalofugal pathway, stria terminalis, and the medial forebrain bundle. Most subcortical fibers pass through the EC initially before breaking into separate bundles, including those that travel to the striatum, or enter the internal capsule.

\section{vmPFC and $\mathrm{mOFC}$}

Axons in the medial bundle from both the vmPFC and mOFC travel through the UF to terminate in multiple vmPFC areas. Other axons from vmPFC and mOFC leave the UF to join the medial forebrain bundle (Fig. 1a,b). The medial forebrain bundle splits into fibers that terminate in the preoptic region and hypothalamus, enter the fornix, or sweep ventral and lateral into the amygdalofugal pathway to terminate in the amygdala (Fig. $2 b$ ). The dorsal bundle travels through the UF, with some fibers terminating in the dorsal vmPFC. Other axons continue dorsally in the UF to merge with fibers of the corpus callosum, cingulum bundle, and superior longitudinal fasciculus. Axons from anterior, medial regions travel posteriorly through the UF to reach the corpus callosum and cross to the contralateral hemisphere. The lateral bundle splits almost immediately into dorsal and lateral groups (Fig. 1a,b). Axons in the dorsal group travel through the EC to terminate in the striatum, enter the IC, or continue in the EC to the claustrum. The lateral group follows the UF before splitting into UF fibers traveling to the lateral OFC and temporal cortex and fibers that enter the EmC that terminate in the insula.

Axons from both the vmPFC and mOFC enter the IC ventrally. As they travel posteriorly, fibers move dorsally within small fascicles that are embedded within the VS and anterior commissure (Fig. $2 c, d$ ). Within the IC, a dorsal group of vmPFC fibers crosses through the AC, sweeping through the inferior thalamic peduncle to the midline and the medial dorsal thalamic nuclei. Those en route to the brainstem (periaqueductal gray, midbrain, and dorsal pons), however, travel in the fascicles ventral to the AC. At the level of the AC, a small group of ventral vmPFC fibers leaves the IC and enters the stria terminalis, providing an additional pathway to the amygdala. mOFC fibers en route to the thalamus travel dorsal to the vmPFC thalamic fibers, passing through or just dorsal to the AC. Axons traveling to the brainstem pass through or ventral to the AC. In addition, some fibers from the mOFC also descend into the pontine nucleus, whereas axons 

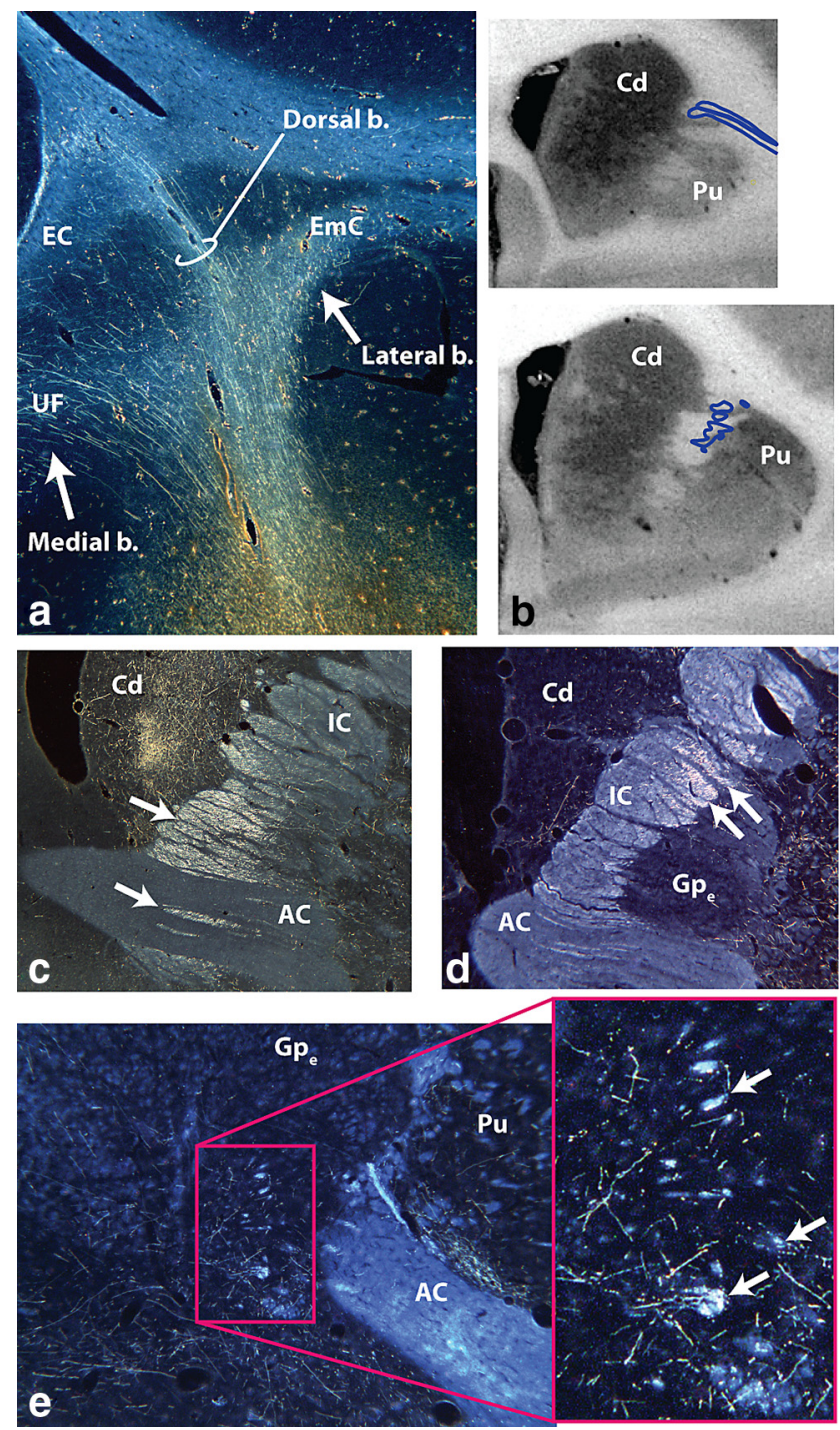

Figure 3. Photomicrographs of $\mathrm{COFC}$ and IOFC pathways. $\boldsymbol{a}, \mathrm{IOFC}$ fibers dividing into medial (UF), dorsal, and lateral bundles. Fibers in the dorsal bundle pass directly through the frontal white matter to reach the corpus callosum and superior longitudinal fasciculus or enter the external or extreme capsules. $\boldsymbol{b}$, Fibers from the IOFC enter the IC dorsolaterally. $c$, Fibers from the $\mathrm{COFC}$ pass through the anterior commissure to the brainstem; those dorsal to the commissure terminate in the thalamus. $\boldsymbol{d}$, All descending IC IOFC axons are located dorsal to the anterior commissure. Note the relative lateral position compared with the COFC fibers. $e$, Axons from the IOFC form small fascicles (arrows) that leave the external capsule travel medial and enter the amygdalofugal pathway or terminate in the hypothalamus. AC, Anterior commissure; b., bundle; $\mathrm{Cd}$, caudate nucleus; EC, external capsule; EmC, extreme capsule; $G_{\mathrm{e}}$ g globus pallidus, external segment; IC, internal capsule; Pu, putamen; UF, uncinate fasciculus.

from vmPFC do not. Those fibers are located between mOFC thalamic and brainstem axons in the IC.

In summary, projections from vmPFC and mOFC follow similar routes with three notable exceptions: (1) vmPFC fibers within the IC and corpus callosum travel ventrally to those from mOFC within the same structure; (2) mOFC sends fibers into the pontine nucleus, but the vmPFC does not; and (3) some vmPFC fibers traveling to the amygdala enter the stria terminalis, but the mOFC fibers do not.

\section{cOFC and 1OFC}

Like those from vmPFC and $\mathrm{mOFC}, \mathrm{cOFC}$ and $\mathrm{lOFC}$ fibers also use the medial bundle through the UF to innervate other vPFC regions (Fig. 1c,d) and the dorsal bundle to reach the corpus callosum, cingulum bundle, and the superior longitudinal fasciculus. However, the dorsal bundle that carries fibers from these cortical areas does not travel within the UF to reach these WM tracts. Rather, it cuts directly through the UF and travels in the overlying frontal WM before turning medially to the corpus callosum, cingulum bundle, or the superior longitudinal fasciculus (Figs. 1c, 3a). An important exception is axons from the posterior cOFC. These fibers must travel either medially or laterally within the UF to circumvent the lateral ventral ventricle and striatum. Thus, fibers from the medial posterior cOFC travel medially within the UF to follow the trajectory of mOFC fibers to the cingulum bundle and corpus callosum. In contrast, axons from lateral posterior $\mathrm{COFC}$ regions travel in the opposite direction to arch laterally around the striatum.

cOFC and lOFC fibers travel through the EC and enter the IC laterally (Fig. $3 b$ ). Within the IC, axons to the thalamus are dorsal to those terminating in the brainstem. cOFC fibers are located ventral and somewhat medial to $1 \mathrm{OFC}$ axons (Figs. $1 c, d, 3 c, d$ ). As fibers travel posteriorly, axons from both regions move ventrally such that, at the level of the AC, cOFC thalamic fibers are just dorsal to the $\mathrm{AC}$, with its brainstem axons positioned ventrally, some of which are embedded within the AC (Figs. 1c, 3c). In contrast, both thalamic and brainstem lOFC fibers travel dorsal to the AC (Figs. $1 d, 3 d$ ).

Unlike axons from the vmPFC and mOFC, cOFC and lOFC fibers travel in the EC for some distance posteriorly before forming small fascicles. These fascicles travel medially through the putamen and globus pallidus to take a position ventral and lateral to those already within the IC (Fig. 3e). Fibers from these fascicules continue to the subthalamic nucleus, midbrain, and dorsal pons. In some cases, a few fibers reach dorsal brainstem regions by a small bundle of fibers traveling through the thalamus. In addition, some small bundles enter the ventral amygdalofugal pathway or terminate in the hypothalamus. Finally, a group of axons continues in the EC to the claustrum. Axons in the lateral bundle from both the $\mathrm{COFC}$ and IOFC include the UF fibers traveling to the temporal cortex and those that enter the EmC to terminate in the insula (Fig. $1 c, d$ ).

\section{Rules of organization}

The uncinate fasciculus. Although the UF is known for its frontotemporal lobe connection (Schmahmann and Pandya, 2006; Petrides and Pandya, 2007), these frontotemporal axons do not form a distinct bundle within the ventral plate of the vPFC. Rather, fibers that pass through here also connect distal regions of the vPFC. Thus, fibers from each cortical region travel anteroposteriorly and mediolaterally through this WM tract, terminating throughout the vPFC. In addition, vPFC axons use this bundle as a conduit for channeling fibers to other WM tracts. For example, vmPFC and mOFC fibers travel for some distance through the UF to reach the corpus callosum, cingulum bundle, and superior longitudinal fasciculus (Figs. 1, 4). Thus, the UF contains three components: connections between the vPFC and temporal lobe, between distal parts of vPFC regions, and between the vPFC and other WM bundles. Although these three components are intertwined at the base of the vPFC, the projection to the temporal cortex is most isolated laterally as the fiber bundle turns into the temporal lobe.

Internal capsule. Axons from the vPFC occupy the most ventral part of the rostral anterior limb of the IC. Importantly, this includes the small fascicules embedded within the VS itself and fibers running ventral to and through the anterior commissure perpendicularly (Figs. $2 d, 3 c$ ). Fibers within the IC are organized 

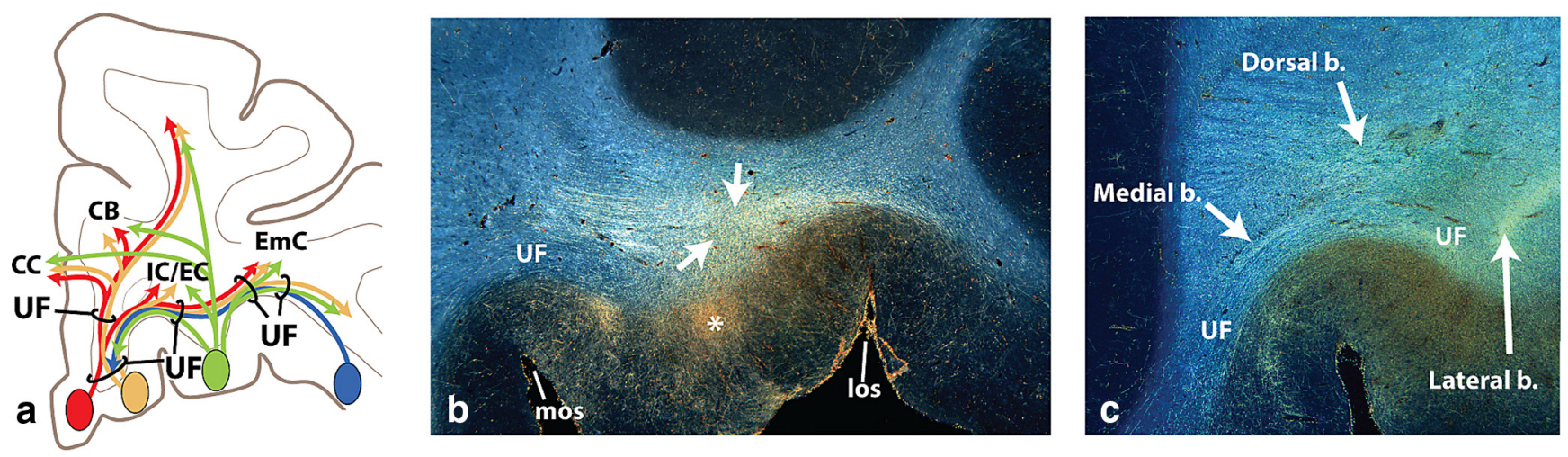

Figure 4. Schematic and photomicrographs illustrating the complexity of the uncinate fasciculus. $\boldsymbol{a}$, Schematic illustrating vPFC axons crossing through the UF to reach other fiber tracts. Note that the medial and central parts of the UF have more crossing fibers compared with the lateral part of the tract. $\boldsymbol{b}-\boldsymbol{c}$, Axons from a $\mathrm{COFC}$ injection site $\left(^{*}\right)$ enter the UF (b), travel a few millimeters and split into different bundles (c). Note the crossing of many fibers as the axons enter the UF in $\boldsymbol{b}$ (arrows). b., Bundle; (B, cingulum bundle; CC, corpus callosum; EC, external capsule; EmC, extreme capsule; IC, internal capsule; los, lateral orbital sulcus; mos, medial orbital sulcus; UF, uncinate fasciculus.
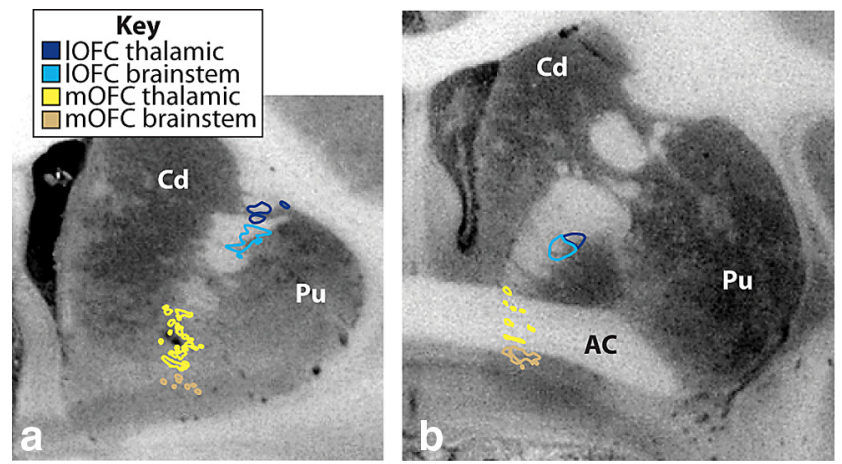

Figure 5. Coronal sections illustrating the different positions of thalamic versus brainstem mOFC fibers (yellow-tan) and IOFC (dark blue-light blue) entering and traveling through the IC. Brainstem fibers (tan and light blue) travel ventral to thalamic fibers (yellow and dark blue). AC, Anterior commissure; Cd, caudate nucleus; IOFC, lateral orbital frontal cortex; m0FC, medial orbital frontal cortex; Pu, putamen.

according to destination. Overall, thalamic IC fibers from each cortical region travel dorsal to their brainstem axons (Fig. 5). Moreover, corticopontine fibers are dorsal to those terminating in the subthalamic nucleus, midbrain, and medulla fibers, but they are ventral to corticothalamic axons. Thus, there is a dorsoventral IC organization from each vPFC area: the dorsal fibers en route to the thalamus, followed by pontine fibers, with the most ventral group traveling to the other brainstem regions. The latter form small bundles, some of which initially course through the VS and globus pallidus before joining the IC (Fig. 3e).

$\checkmark P F C$ position. The medial/lateral position within the vPFC dictates both the route that fibers take to enter major WM tracts as well as the position they take within some of those tracts. Both vmPFC and mOFC fibers travel in the medial bundle to the amygdalofugal pathway via the medial forebrain bundle. In contrast, the more lateral OFC regions first use the dorsal bundle to enter the EC and then form fascicules that are embedded within the basal forebrain, some of which enter the amygdalofugal pathway. Only axons from the vmPFC use the stria terminalis as an additional pathway to the amygdala and the bed nucleus stria terminalis.

Fibers from the vmPFC and mOFC travel for some distance through the UF to reach the corpus callosum and cingulum bundle. In contrast, the more lateral regions cut directly across the UF to travel in the frontal WM to reach these bundles (Figs. 2a, 3a,4). vPFC axons also enter the IC with a medial to lateral topography.
The vmPFC and mOFC fibers enter the IC (and striatum) ventrally, directly through the subcaudate $\mathrm{WM}$, whereas the cOFC and IOFC pass through the EC and enter the IC from a more lateral and dorsal position. The vmPFC and mOFC fibers move dorsal through the IC as they travel posteriorly. In contrast, fibers from the more lateral cortical regions move ventrally (Fig. $2 c, 3 b$ ).

Within specific WM pathways, the medial/lateral cortical position also dictates the position within the tract. Fibers from all vPFC areas enter the internal capsule anteriorly and follow a medial/lateral, ventral/dorsal topography. Thus, fibers from medial areas travel ventral to axons from lateral vPFC regions (Fig. 6). Axons from vmPFC regions remain within small fascicules embedded within ventral striatum or AC. In contrast, axons from the $\mathrm{COFC}$ and IOFC are located dorsally in the IC. Thus, fibers are stacked in the internal capsule with the vmPFC axons ventral or embedded within the $\mathrm{AC}$ and the lateral OFC regions positioned dorsal to the AC. This topography is maintained (albeit with a great deal of compression) as they enter the inferior thalamic peduncle.

Axons traveling in the corpus callosum are organized similarly. Axons from more medial vPFC areas travel ventral to those fibers originating in lateral regions. Thus, vmPFC and mOFC fibers are ventral to cOFC, etc. (Fig. 7). However, in most association WM tracts, such as the cingulum bundle or the UF, it was difficult to discern a topographic arrangement. Thus, axons from different vPFC regions appeared to overlap extensively as they travel to their destinations.

In summary, important characteristics and rules that govern vPFC fiber trajectories are as follows: (1) vPFC fibers travel primarily in the amygdalofugal pathway to reach the amygdala. (2) vPFC efferent fibers to subcortical thalamic and brainstem targets pass through small fascicles embedded in the VS and anterior commissure, suggesting that these bundles are part of the IC. Moreover, within the IC, there is a topographic organization of thalamic and brainstem fibers from each vPFC region, such that each set of descending fibers occupies a specific position within the IC. (3) Importantly, it is not possible to separate a specific frontal-temporal set of fibers from the axons connecting the vPFC or those that join other WM bundles with the exception of the most lateral part. Thus, the vPFC uses the UF to connect not only the vPFC-temporal cortices, but also vPFC-vPFC areas and as a conduit to reach other WM tracts. (4) Finally, the medial/ lateral position of the $\mathrm{vPFC}$ fiber origin affects the route those 
efferents take to their terminals, allowing predictions about where fibers from each area likely travel.

Deep brain stimulation sites. The most effective SCGwm contacts ( 1 and 2) are at the border between the SCG and the inferior rostral gyrus (Hamani et al., 2009). Contact 1 is within the inferior rostral gyrus, contact 2 is within the SCG, and contracts 0 and 3 are ventral and dorsal, respectively. Placing the electrode representation in the same position in the $3 \mathrm{D}$ monkey model shows that contacts $0-2$ involve (1) all connections from vmPFC areas adjacent to the electrode contacts (both cortical and subcortical projections) (Fig. 8a), (2) UF fibers from nonadjacent vmPFC and $\mathrm{mOFC}$ as they travel medially to other vPFC areas and/or enter the medial forebrain bundle (Fig. 8b), (3)

a subset of cOFC fibers traveling medially to innervate medial PFC areas (Fig. 8b), (4) axons traveling from the contralateral vmPFC and mOFC (not illustrated), and (5) a subset of anterior vmPFC and $\mathrm{mOFC}$ en route to the corpus callosum through the UF. Contact 3 involves primarily fibers in the corpus callosum. In addition, this site captures a subset of fibers traveling from the $\mathrm{mOFC}$ and posterior $\mathrm{COFC}$ to the cingulum bundle and superior longitudinal fasciculus.

The VC/VS electrode is implanted at an angle, positioning contact 0 most posteriorly. Each contact activates a different subset of corticothalamic and brainstem fibers (Fig. $8 c-f$ ). Axons from the vmPFC and mOFC pass through contact 0 , most traveling to the brainstem, with few to the thalamus (Fig. $8 c, d$ ). In contrast, contact 1 captures fibers from the vmPFC traveling to the thalamus, but not those to the brainstem. Contact 1 involves some cOFC brainstem axons, but few thalamic cOFC fibers (Fig. $8 e$ ). Contact 2 captures more mOFC thalamic fibers, but none traveling to the brainstem. cOFC brainstem fibers also pass through this site (Fig. 8e). Contact 3 captures both brainstem and thalamic cOFC fibers and brainstem fibers from $1 \mathrm{OFC}$, but few, if any, of the vmPFC, mOFC, or lOFC thalamic fibers (Fig. $8 f$ ).

DBS field modeling reveals additional important detail about pathways likely to be captured by DBS. Stimulation parameters scaled to $40 \%$ of those in humans give an effective stimulation diameter of $3.10 \mathrm{~mm}$ (Fig. $8 g-r$ ). Contact 0 captures fibers traveling to the thalamus from both the vmPFC and mOFC (Figs. $8 g, h)$. Contact 1 includes some cOFC thalamic fibers and a few lOFC brainstem fibers (Figs. $8 i-l$ ). At contact 2, some fibers from the vmPFC and more of those from the $\mathrm{mOFC}$ and $\mathrm{COFC}$ traveling to the thalamus will be included along with fibers from the brainstem component from the $\mathrm{mOFC}$ and the lOFC (Figs. $8 \mathrm{~m}-$ p). Finally, contact 3 captures most cOFC thalamic fibers and some brainstem axons (Figs. 8q,r). In addition, some mOFC thalamic fibers are captured and both thalamic and brainstem lOFC axons.

Finally, contact 0 at the nucleus accumbens site is placed in the shell of the VS and contact 1 in the core. In contrast to the SCG and VC/VS, stimulation of the ventral 2 contacts is located primarily in gray matter, but within corticostriatal fibers. Contacts 2 and 3 are within the $\mathrm{VC}$ and likely involve similar cortical connections as those described above for the VC/VS target.
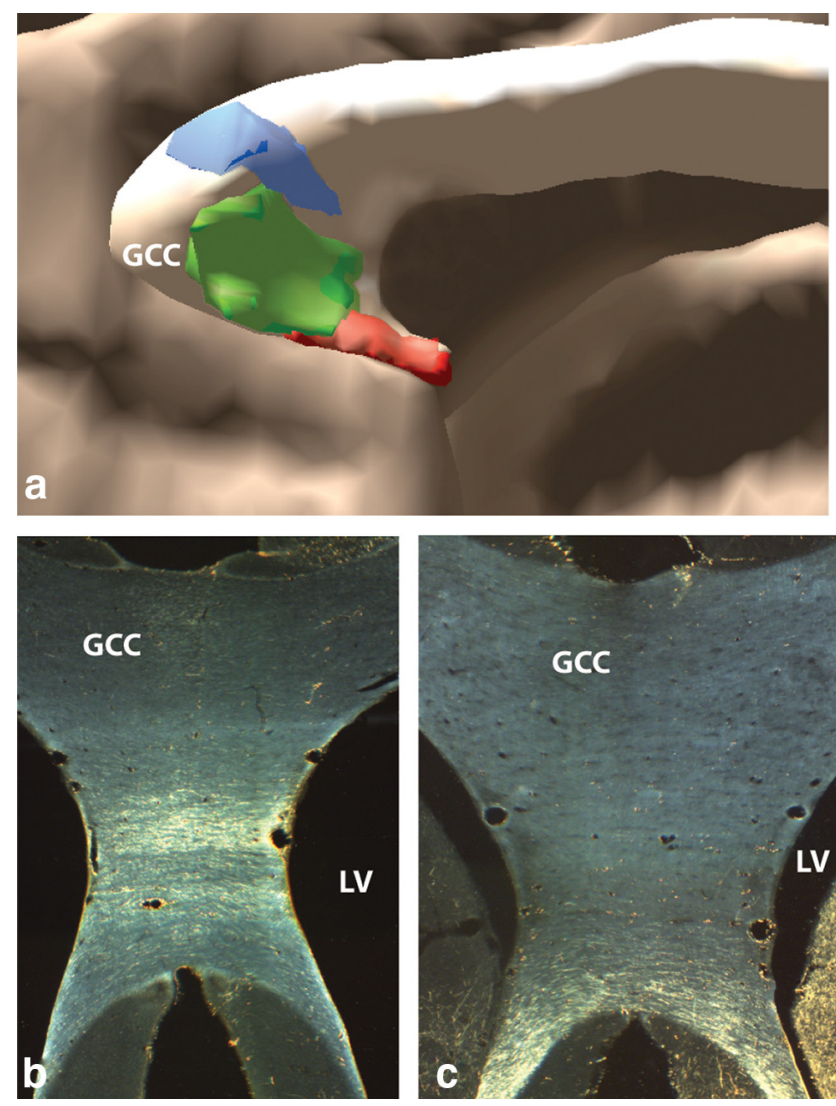

Figure 7. Organization of $\mathrm{VPFC}$ pathways in the corpus callosum. $\boldsymbol{a}$, Sagittal view of 3D model of $\mathrm{VPFC}$ pathways in corpus callosum. Fibers originating from more lateral vPFC regions (blue) cross dorsally to fibers from more medial vPFC areas (red). $\boldsymbol{b}$, Micrograph of COFC fibers crossing mid-level genu of corpus callosum. $c$, Micrograph of vmPFC crossing most ventral portion of corpus callosum. GCC, Genu of corpus callosum; LV, lateral ventricle; blue, IOFC; green, COFC; red, vmPFC.

\section{Discussion}

Using conventional tracing techniques coupled with 3D pathway reconstructions, we identified key characteristics of trajectories of the two main vPFC cortical and subcortical bundles, the UF and IC. Specifically, the UF connects not only the vPFC with the temporal lobe, but also different vPFC regions and, indirectly, the 

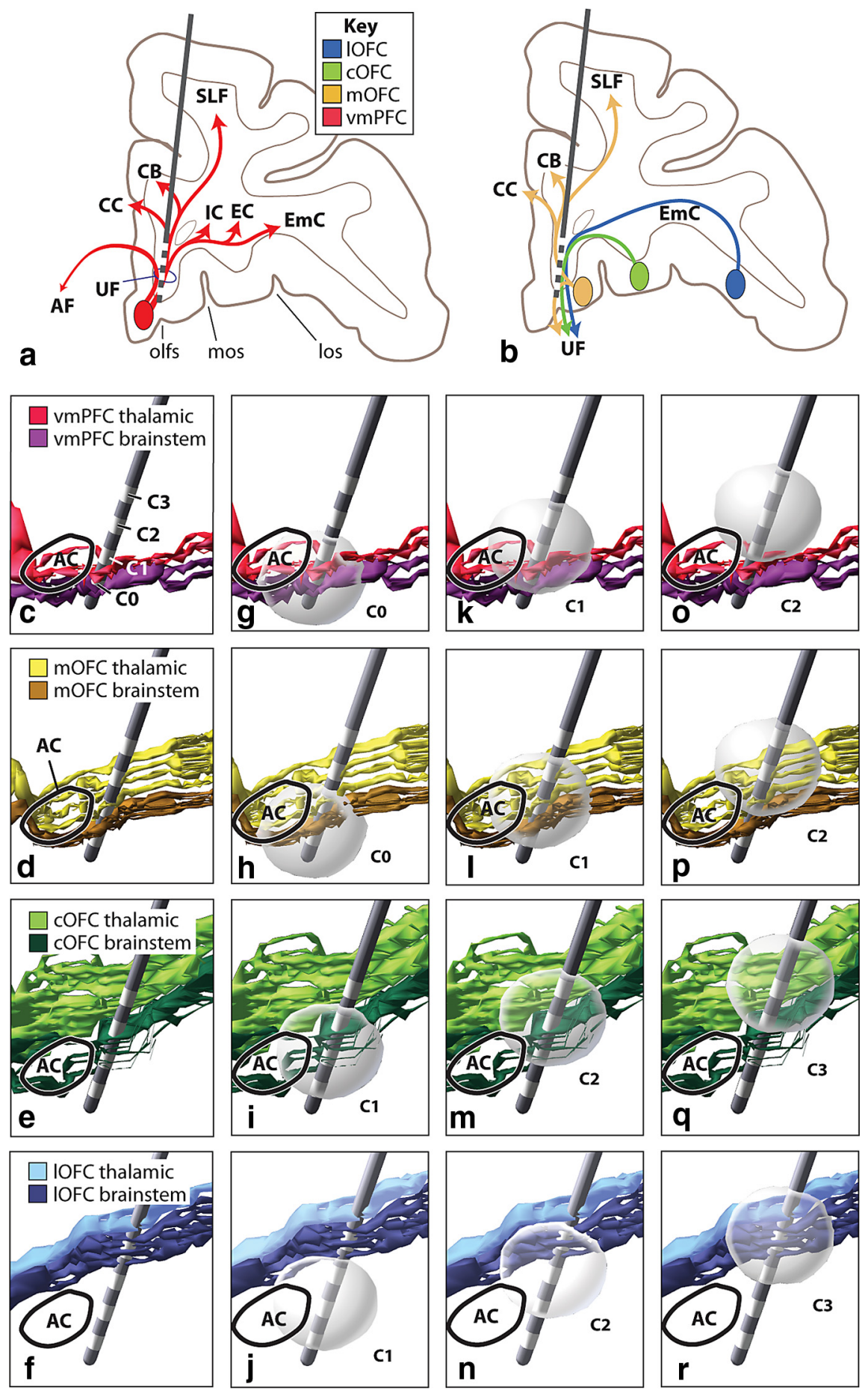

Figure 8. Modeled DBS electrodes at the SCGwm andVC/NStargets. $\boldsymbol{a}$, TheSCGwm target involves all fibers from cortical areas adjacent to the electrode, including descending projections. $\boldsymbol{b}$, SCGwm target also involves other VPFC fibers that pass through the site, including axons from lateral vPFC regions traveling medially and those from medial OFC areas traveling dorsal. $\mathbf{c}-\boldsymbol{f}$, Sagittal view of specific ventral PFC bundles traveling in the IC with an electrode representation embedded at the VC/NS site. Each contact captures a different set of thalamic and/or brainstem fibers. $\boldsymbol{g}-\boldsymbol{r}$, Spheres indicating volume of tissue activated by electrical stimulation of a pulse width of $60 \mu \mathrm{s}$ and $3 \mathrm{~V}$ at each contact site. The electrode field model is not more oval shaped because we used the isotropic simplification that allowed us to move the electrode to any position. $\mathrm{AF}$, Ventral amygdalofugal bundle; $\mathrm{AC}$, anterior commissure; $\mathrm{CO}-\mathrm{C}$, contacts $0-3$; $\mathrm{CB}$, cingulum bundle; $\mathrm{CC}$, corpus callosum; $\mathrm{COFC}$, central orbital prefrontal cortex; EC, external capsule; EmC, extreme capsule; IC, internal capsule; IOFC, lateral orbital prefrontal cortex; los, lateral orbital sulcus; mOFC, medial orbital prefrontal cortex; mos, medial orbital sulcus; olfs, olfactory sulcus; SLF, superior longitudinal fasciculus; UF, uncinate fasciculus; vmPFC, ventral medial prefrontal cortex.

vPFC with the dorsal PFC by merging with other WM bundles. The medial/lateral cortical vPFC position dictates specific trajectories fibers take to reach their cortical and subcortical targets and the position of fibers within certain bundles. Together, these data demonstrate how the main vPFC WM bundles are segmented and the paths fibers use to reach their targets.

\section{UF and IC Characteristics}

The WM bundle that runs in the ventral plate of the $\mathrm{vPFC}$, and that is typically labeled as the UF, contains three components, the vPFC-temporal lobe connections, the $\mathrm{vPFC}-\mathrm{vPFC}$ connections, and it also serves as a conduit for $\mathrm{vPFC}$ fibers to join other tracts. These results support early dissection and degeneration studies (Dejerine, 1895; Nauta, 1964). The central and medial parts of this bundle contain a large proportion of fibers that link the vPFC areas that mediate different aspects of reward processing (O'Doherty et al., 2001; Rudebeck et al., 2008). In contrast, fibers connecting $\mathrm{vPFC}$ to the temporal lobe are most isolated laterally. Lesions placed here therefore are less likely to affect reward processing, as the majority of vPFC-vPFC connections will not be involved (Gutnikov et al., 1997; Browning and Gaffan, 2008). Axons also travel through the UF, merging into the cingulum bundle, and the superior longitudinal fasciculus to reach the dorsal PFC. The human UF is also likely to contain these components. Fibers from all $\mathrm{vPFC}$ regions also must pass through the UF to reach distal $\mathrm{NPFC}$ regions or join other pathways. Although $\mathrm{COFC}$ fibers anterior to the striatum cross directly through the UF, at posterior levels, these fibers must travel some distance either medially or laterally within the UF to circumvent the striatum to reach the corpus callosum and cingulum bundle.

Although some axons reach the anterior amygdala through the UF (Petrides and Pandya, 2007), consistent with its reciprocal amygdalo-vPFC connection (Nauta, 1961; Amaral et al., 1992), a main route is via the amygdalofugal pathway. This pathway, not commonly outlined in human atlases, is located within the basal forebrain sweeping dorsally from the amygdala and traveling ventrally to the pallidal complex (Kim and Whalen, 2009). Our experiments suggest that this pathway also carries the reciprocal vPFC projections to the amygdala in humans. Note that axons from the vmPFC use the stria terminalis as an additional pathway to the amygdala.

Within the IC, vPFC thalamic fibers from each cortical region travel dorsally to those terminating in the brainstem. This suggests that axons from different cortical layers remain separate in the capsule. vPFC fibers pass through the ventral-most part of the anterior IC. The border here is classically defined as the dorsal 

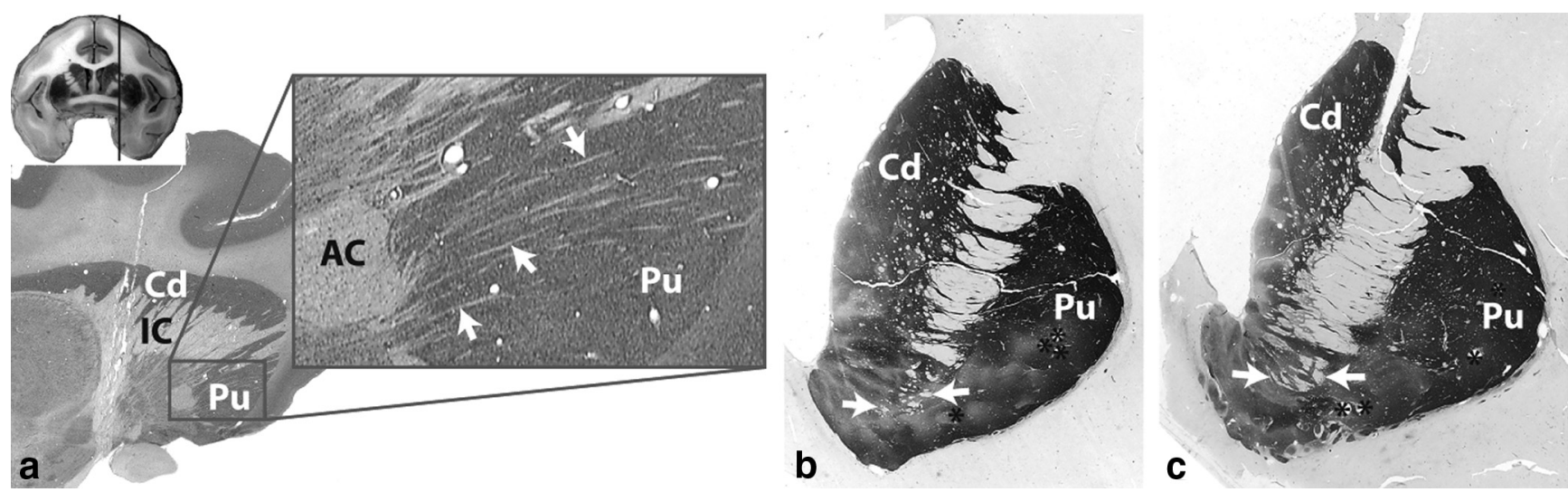

Figure 9. White matter fascicules through the ventral forebrain. $\boldsymbol{a}$, Nissl-stained sagittal section through the monkey brain. Arrows indicate white matter tracts from the vPFC. $\boldsymbol{b}$ - $\boldsymbol{c}$, Acetylcholinesterase-stained coronal sections through the human striatum at different rostral/caudal levels. Arrows indicate WM fascicules passing through the ventral striatum. AC, Anterior commissure; Cd, caudate; IC, internal capsule; Pu, putamen. Asterisks indicate blood vessels.

nucleus accumbens and AC (Dejerine, 1895; Schmahmann and Pandya, 2006). However, a significant proportion of the descending vPFC fibers travels in the WM fascicules embedded within accumbens and anterior commissure. These fascicules thus compose an integral part of the IC (Fig. 9a). Small fascicules are also embedded in the human VS (Dejerine, 1895) (Fig. 9b) and are likely to carry the descending vPFC IC fibers.

\section{Medial/lateral $v P F C$ position of origin dictates fiber trajectories}

Axons from medial areas reach the corpus callosum, cingulum bundle, and superior longitudinal fasciculus via a dorsal route, first traveling in the UF. Although axons from lateral OFC regions also reach those bundles traveling dorsally, they only cut

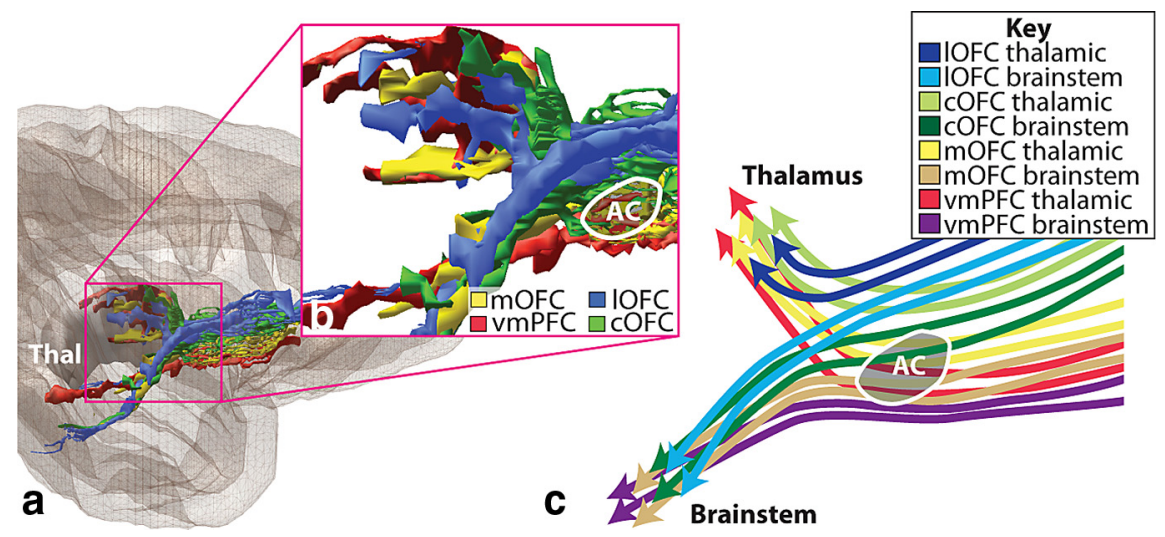

Figure 10. A parasagittal section illustrating the relationship between brainstem and thalamic internal capsule fibers from the different VPFC regions. $\boldsymbol{a}$, Overview of VPFC fibers in the IC. $\boldsymbol{b}$, Enlargement of the anterior internal capsule showing the split of thalamic and brainstem fibers caudal to the anterior commissure. $c$, Schematic of the separation of thalamic and brainstem fibers. Thalamic fibers from vmPFC (red) occupy a similar position as brainstem fibers from $\mathrm{mOFC}$ (orange); $\mathrm{mOFC}$ thalamic fibers (yellow) occupy a similar position as brainstem fibers from c $\mathrm{OFC}$ (dark green). $\mathrm{AC}$, Anterior commissure; $\mathrm{COFC}$, central orbital frontal cortex; mOFC, medial orbital frontal cortex; IOFC, lateral orbital frontal cortex; Thal, thalamus; vmPFC, ventral medial prefrontal cortex.

through the UF, but do not travel through

it. cOFC fibers take different routes depending on their anteriorposterior position. To reach the amygdalofugal pathway, vmPFC and $\mathrm{mOFC}$ areas send fibers through the medial forebrain bundle, whereas more lateral areas send axons through small fascicles that leave the EC. Within particular bundles, axons are also organized with respect to their medial/lateral cortical origin. Medial vPFC fibers in the corpus callosum are located most ventrally, and axons from the lOFC are most dorsal. In contrast, the UF and cingulum bundles do not appear to be organized in this way.

The IC has a similar medial/lateral organization, with vmPFC fibers positioned most ventrally, followed in ascending order by mOFC, cOFC, and lOFC projections. Superimposed on this topographic organization is the arrangement of thalamic versus brainstem axons from each cortical area. This creates a complex convergence between thalamic and brainstem fibers from different vPFC regions. vmPFC and mOFC brainstem fibers are embedded within the VS; the fibers that pass through the AC head for the thalamus. In contrast, COFC axons within the AC travel to the brainstem, and those dorsal to the AC travel to the thalamus. Thus, the medial/lateral origin of vPFC fibers, coupled with their thalamic versus brainstem organization, positions thalamic IC fibers from medial vPFC regions with brainstem fibers from more lateral vPFC areas (Fig. 10). These results imply that lesions or DBS at various IC locations will affect different combinations of thalamic and brainstem vPFC fibers.

\section{Implications for human tractography}

Although significant advances in diffusion tractography have been made that address technical problems, such as the difficulty of navigating through crossing fibers and the level of resolution available (Behrens et al., 2007; Jones, 2008), these issues have not been completely resolved. It is therefore critical to know which connections observed with DTI correspond to experimental observations. All vPFC axons travel some distance within the UF (or cut through it) before separating into different bundles. Thus, a complex mixture of differently oriented axons travels through the UF. However, laterally, where the UF curves into the temporal cortex, most fibers connect the vPFC and temporal lobe. Here, DTI will be more reliable because of higher anisotropy (Croxson et al., 2005). In contrast, tractography from a seed placed in the vmPFC cannot accurately identify all fibers passing through the area, including OFC fibers, fibers traveling to the dorsal PFC, and the axons to several brainstem regions (Gutman et al., 2009).

The anterior limb of the human IC carries primarily prefrontal fibers, with motor- and sensory-associated fibers traveling in 
the posterior limb (Beevor and Horsley, 1890). Our data show that vPFC fibers are carried in the ventral-most part, including fascicles embedded in the gray matter. These primarily connect to the medial dorsal nucleus of the thalamus, but not in the motor or sensory thalamic nuclei (Ray and Price, 1993; McFarland and Haber, 2002). DTI tractography, using experimental data as guidance, shows this thalamic connection from the dorsal and lateral PFC regions, with fibers located in the IC dorsal to the AC (Klein et al., 2010). In contrast, vPFC DTI studies in humans demonstrate widespread connections to the entire thalamus and brainstem, including motor and sensory areas (Gutman et al., 2009). These "false positives" likely are the result of the inability to accurately follow fibers through the ventral part of the anterior limb of the IC. vPFC thalamic and brainstem fibers pass through here, some in relatively small, scattered fascicules, but the voxel size of these fascicules is smaller than the available resolution of DTI scans. In summary, our data provide a guide for where to expect vPFC fibers to run, helping identify false positives and negatives resulting from DTI tractography. It also provides the foundation for the required resolution parameters that will work best for specific WM tracts.

\section{What gets stimulated at the DBS targets?}

Effectiveness of DBS for depression at the SCGwm and VC/VS (WM) sites has not been directly compared with respect to patient selection criteria. Nonetheless, both sites as well as the $\mathrm{VC} / \mathrm{VS}$ site for obsessive-compulsive disorder are effective in over $50 \%$ of otherwise intractable patients (Greenberg et al., 2010b; Kennedy et al., 2011). Stimulation at the SCGwm site captures all cortical and subcortical projections from the area surrounding each contact site. However, it also captures fibers from nonadjacent cortical areas passing through the target, including connections between different vPFC areas, and OFC fibers traveling to corpus callosum, medial forebrain bundle, cingulum bundle, and superior longitudinal fasciculus. In addition, this target captures the extensive brainstem connections from the subgenual cingulate gyrus. In contrast, neither the VS nor the VC/VS site directly involves corticocortical fibers. Each contact in the VC/VS site involves a different combination of thalamic and/or brainstem bundles. For example, contact 0 essentially limits stimulation to brainstem bundles, and contact 1 likely involves thalamic fibers from the medial areas. Contact 2 captures different combinations of thalamic and brainstem fibers. When electric field models are incorporated, contacts recruit a larger subset of projections. Nonetheless, they remain distinct. Of particular importance is that the three DBS targets capture subsets of fibers that include both thalamic and brainstem fibers. Thus, an important part of the clinical effectiveness of DBS is likely to require a combination of thalamic and brainstem fibers. In summary, these data allow comparative assessment of the connections associated with clinical outcomes at different DBS targets (Gutman et al., 2009; Greenberg et al., 2010a).

\section{References}

Amaral DG, Price JL, Pitkanen A, Carmichael ST (1992) Anatomical organization of the primate amygdaloid complex. In: The amygdala: neurobiological aspects of emotion, memory, and mental dysfunction (Aggleton JP, ed), pp 1-66. New York: Wiley.

Beckmann M, Johansen-Berg H, Rushworth MF (2009) Connectivity-based parcellation of human cingulate cortex and its relation to functional specialization. J Neurosci 29:1175-1190.

Beevor CE, Horsley V (1890) An experimental investigation into the arrangement of the excitable fibres of the internal capsule of the bonnet monkey (Macacus sinicus). Philos Trans R Soc Lond B Biol Sci 181:49-88.
Behrens TE, Berg HJ, Jbabdi S, Rushworth MF, Woolrich MW (2007) Probabilistic diffusion tractography with multiple fibre orientations: What can we gain? Neuroimage 34:144-155.

Browning PG, Gaffan D (2008) Impairment in object-in-place scene learning after uncinate fascicle section in macaque monkeys. Behav Neurosci 122:477-482.

Chamberlain SR, Menzies L, Hampshire A, Suckling J, Fineberg NA, del Campo N, Aitken M, Craig K, Owen AM, Bullmore ET, Robbins TW, Sahakian BJ (2008) Orbitofrontal dysfunction in patients with obsessive-compulsive disorder and their unaffected relatives. Science 321:421-422.

Chase HW, Clark L, Myers CE, Gluck MA, Sahakian BJ, Bullmore ET, Robbins TW (2008) The role of the orbitofrontal cortex in human discrimination learning. Neuropsychologia 46:1326-1337.

Croxson PL, Johansen-Berg H, Behrens TE, Robson MD, Pinsk MA, Gross CG, Richter W, Richter MC, Kastner S, Rushworth MF (2005) Quantitative investigation of connections of the prefrontal cortex in the human and macaque using probabilistic diffusion tractography. J Neurosci 25:8854-8866.

Cullen KR, Klimes-Dougan B, Muetzel R, Mueller BA, Camchong J, Houri A, Kurma S, Lim KO (2010) Altered white matter microstructure in adolescents with major depression: a preliminary study. J Am Acad Child Adolesc Psychiatry 49:173-183.e1.

Dejerine J (1895) Anatomie des centres nerveux. Paris: Rueff.

Greenberg BD, Rauch SL, Haber SN (2010a) Invasive circuitry-based neurotherapeutics: stereotactic ablation and deep brain stimulation for OCD. Neuropsychopharmacology 35:317-336.

Greenberg BD, Gabriels LA, Malone DA Jr, Rezai AR, Friehs GM, Okun MS, Shapira NA, Foote KD, Cosyns PR, Kubu CS, Malloy PF, Salloway SP, Giftakis JE, Rise MT, Machado AG, Baker KB, Stypulkowski PH, Goodman WK, Rasmussen SA, Nuttin BJ (2010b) Deep brain stimulation of the ventral internal capsule/ventral striatum for obsessive-compulsive disorder: worldwide experience. Mol Psychiatry 15:64-79.

Gutman DA, Holtzheimer PE, Behrens TE, Johansen-Berg H, Mayberg HS (2009) A tractography analysis of two deep brain stimulation white matter targets for depression. Biol Psychiatry 65:276-282.

Gutnikov SA, Ma YY, Buckley MJ, Gaffan D (1997) Monkeys can associate visual stimuli with reward delayed by $1 \mathrm{~s}$ even after perirhinal cortex ablation, uncinate fascicle section or amygdalectomy. Behav Brain Res 87:85-96.

Haber SN, Kim KS, Mailly P, Calzavara R (2006) Reward-related cortical inputs define a large striatal region in primates that interface with associative cortical inputs, providing a substrate for incentive-based learning. J Neurosci 26:8368-8376.

Hamani C, Mayberg H, Snyder B, Giacobbe P, Kennedy S, Lozano AM (2009) Deep brain stimulation of the subcallosal cingulate gyrus for depression: anatomical location of active contacts in clinical responders and a suggested guideline for targeting. J Neurosurg 111:1209-1215.

Johansen-Berg H, Gutman DA, Behrens TE, Matthews PM, Rushworth MF, Katz E, Lozano AM, Mayberg HS (2008) Anatomical connectivity of the subgenual cingulate region targeted with deep brain stimulation for treatment-resistant depression. Cereb Cortex 18:1374-1383.

Jones DK (2008) Studying connections in the living human brain with diffusion MRI. Cortex 44:936-952.

Kennedy SH, Giacobbe P, Rizvi SJ, Placenza FM, Nishikawa Y, Mayberg HS, Lozano AM (2011) Deep brain stimulation for treatment-resistant depression: follow-up after 3 to 6 years. Am J Psychiatry 168:502-510.

Kim MJ, Whalen PJ (2009) The structural integrity of an amygdala-prefrontal pathway predicts trait anxiety. J Neurosci 29:11614-11618.

Klein JC, Rushworth MF, Behrens TE, Mackay CE, de Crespigny AJ, D'Arceuil H, Johansen-Berg H (2010) Topography of connections between human prefrontal cortex and mediodorsal thalamus studied with diffusion tractography. Neuroimage 51:555-564.

Kremer JR, Mastronarde DN, McIntosh JR (1996) Computer visualization of three-dimensional image data using IMOD. J Struct Biol 116:71-76.

Makris N, Kennedy DN, McInerney S, Sorensen AG, Wang R, Caviness VS Jr, Pandya DN (2005) Segmentation of subcomponents within the superior longitudinal fascicle in humans: a quantitative, in vivo, DT-MRI study. Cereb Cortex 15:854-869.

Mayberg HS (2007) Defining the neural circuitry of depression: toward a new nosology with therapeutic implications. Biol Psychiatry 61:729-730.

Mayberg HS, Lozano AM, Voon V, McNeely HE, Seminowicz D, Hamani C, 
Schwalb JM, Kennedy SH (2005) Deep brain stimulation for treatmentresistant depression. Neuron 45:651-660.

McFarland NR, Haber SN (2002) Thalamic relay nuclei of the basal ganglia form both reciprocal and nonreciprocal cortical connections, linking multiple frontal cortical areas. J Neurosci 22:8117-8132.

Miocinovic S, Zhang J, Xu W, Russo GS, Vitek JL, Mclntyre CC (2007) Stereotactic neurosurgical planning, recording, and visualization for deep brain stimulation in non-human primates. J Neurosci Methods 162:3241.

Nauta WJH (1961) Fibre degeneration following lesions of the amygdaloid complex in the monkey. J Anat 95:515-531.

Nauta WJH (1964) Some efferent connections of the prefrontal cortex in the monkey. In: The frontal granular cortex and behavior (Warren J, Akert K, eds), pp 397-409. New York: McGraw-Hill.

O’Doherty J, Kringelbach ML, Rolls ET, Hornak J, Andrews C (2001) Abstract reward and punishment representations in the human orbitofrontal cortex. Nat Neurosci 4:95-102.

O’Doherty J, Critchley H, Deichmann R, Dolan RJ (2003) Dissociating valence of outcome from behavioral control in human orbital and ventral prefrontal cortices. J Neurosci 23:7931-7939.

Petrides M, Pandya DN (2007) Efferent association pathways from the rostral prefrontal cortex in the macaque monkey. J Neurosci 27:11573-11586.

Petrides M, Alivisatos B, Frey S (2002) Differential activation of the human orbital, mid-ventrolateral, and mid-dorsolateral prefrontal cortex during the processing of visual stimuli. Proc Natl Acad Sci U S A 99:5649-5654.

Price G, Cercignani M, Parker GJ, Altmann DR, Barnes TR, Barker GJ, Joyce EM, Ron MA (2008) White matter tracts in first-episode psy- chosis: a DTI tractography study of the uncinate fasciculus. Neuroimage 39:949-955.

Price JL, Drevets WC (2010) Neurocircuitry of mood disorders. Neuropsychopharmacology 35:192-216.

Ray JP, Price JL (1993) The organization of projections from the mediodorsal nucleus of the thalamus to orbital and medial prefrontal cortex in macaque monkeys. J Comp Neurol 337:1-31.

Rudebeck PH, Bannerman DM, Rushworth MF (2008) The contribution of distinct subregions of the ventromedial frontal cortex to emotion, social behavior, and decision making. Cogn Affect Behav Neurosci 8:485-497.

Schlaepfer TE, Cohen MX, Frick C, Kosel M, Brodesser D, Axmacher N, Joe AY, Kreft M, Lenartz D, Sturm V (2008) Deep brain stimulation to reward circuitry alleviates anhedonia in refractory major depression. Neuropsychopharmacology 33:368-377.

Schmahmann J, Pandya D (2006) Fiber pathways of the brain. New York: Oxford UP.

Schmahmann JD, Pandya DN, Wang R, Dai G, D’Arceuil HE, de Crespigny AJ, Wedeen VJ (2007) Association fibre pathways of the brain: parallel observations from diffusion spectrum imaging and autoradiography. Brain 130:630-653.

Volkow ND, Wang GJ, Fowler JS, Logan J, Gatley SJ, Wong C, Hitzemann R, Pappas NR (1999) Reinforcing effects of psychostimulants in humans are associated with increases in brain dopamine and occupancy of $\mathrm{D}(2)$ receptors. J Pharmacol Exp Ther 291:409-415.

Yin D, Valles FE, Fiandaca MS, Forsayeth J, Larson P, Starr P, Bankiewicz KS (2009) Striatal volume differences between non-human and human primates. J Neurosci Methods 176:200-205. 